\title{
Radical treatment for bilateral vertebral artery dissecting aneurysms by reconstruction of the vertebral artery
}

\author{
Nakao Ota, MD, Rokuya Tanikawa, MD, Hirotake Eda, MD, Takashi Matsumoto, MD, \\ Takanori Miyazaki, MD, Hidetoshi Matsukawa, MD, Takeshi Yanagisawa, MD, Go Suzuki, MD, PhD, \\ Shiro Miyata, MD, PhD, Jumpei Oda, MD, PhD, Kosumo Noda, MD, Toshiyuki Tsuboi, MD, PhD, \\ Rihei Takeda, MD, Hiroyasu Kamiyama, MD, and Sadahisa Tokuda, MD \\ Stroke Center, Department of Neurosurgery, Teishinkai Hospital, Sapporo, Japan
}

\begin{abstract}
OBJECTIVE Bilateral vertebral artery dissecting aneurysms (VADAs) have a poor prognosis because progressive enlargement of the aneurysms compresses the brainstem or causes subarachnoid hemorrhage. The trapping of 1 vertebral artery (VA) places increased hemodynamic stress on the contralateral VA and may lead to enlargement and rupture. Therefore, management strategies are controversial. This study describes a radical treatment for bilateral VADAs using bypass surgery.

METHODS Seven patients with bilateral VADAs were included. Three patients were treated by trapping of 1 VA via coiling or clipping at another hospital; the previously treated VA in 1 patient and the contralateral untreated VA in 2 patients subsequently enlarged. The other 4 patients presented without previous intervention and progressive enlargement of the aneurysms.
\end{abstract}

RESULTS The post-coil embolization patients underwent $V_{3}$-posterior cerebral artery (PCA) bypass and trapping. The other 4 patients underwent $V A$ reconstruction via $V_{3}-V_{4}$ or $V_{4}-V_{4}$ bypass, with contralateral trapping on a separate day in 3 patients and observation in 1 patient. Perioperative complications included 1 case of cerebrospinal fluid leakage for which the patient required an additional operation, 1 case of dysphagia and facial palsy due to sigmoid sinus thrombosis, and 1 case of dysphagia. The long-term outcomes of these patients were favorable.

CONCLUSIONS Patients with bilateral VADAs require treatment on both sides. If VA trapping is performed first, the treatment options for the other side are limited to $\mathrm{V}_{3}-\mathrm{PCA}$ bypass and trapping. This procedure is effective; however, it is also invasive and technically difficult. In cases of bilateral VADAs in which it is feasible to reconstruct 1 side, the best approach is to begin by reconstructing the VA that appears technically easiest, followed by trapping of the contralateral VADA. This strategy allows enough time to suture vessels because contralateral reverse flow is maintained.

http://thejns.org/doi/abs/10.3171/2015.8.JNS15362

KEY WORDS bilateral vertebral artery dissecting aneurysm; vascular reconstruction; $V_{3}-V_{4}$ bypass; $V_{3}$-PCA bypass; $\mathrm{V}_{4}-\mathrm{V}_{4}$ bypass; vascular disorders

$\mathrm{P}$ ATIENTS with bilateral vertebral artery dissecting aneurysms (VADAs) have a poor prognosis because progressive enlargement of the aneurysms compresses the brainstem or causes subarachnoid hemorrhage (SAH). ${ }^{11,18,33,36}$ Trapping of the vertebral artery (VA) places increased hemodynamic stress on the contralateral VA and leads to enlargement and possible rupture of that aneurysm. ${ }^{11,13,36}$ Thus, the optimal treatment strategy is controversial. Endovascular treatments, such as stentassisted coiling, the stent-within-a-stent technique, or covered stent graft treatment, are becoming increasingly common and have been reported to have relatively good outcomes. . $^{1,8,19,38,42,44}$ More recently, flow-diverting devices intended to hemodynamically exclude aneurysms from the parent vessel lumen as the primary treatment have been reported to have good outcomes. ${ }^{5,6,24,40}$ However, the long-term outcomes of such endovascular treatments remain unclear, and they are plagued by issues such as instent thrombosis and occlusion of important perforating arteries. ${ }^{4,16,25}$

ABBREVIATIONS AICA = anterior inferior cerebellar artery; $C N=$ cranial nerve; $C T A=C T$ angiography; $\mathrm{mRS}=$ modified Rankin Scale; $\mathrm{OA}=$ occipital artery; $\mathrm{PCA}=$ posterior cerebral artery; PICA = posterior inferior cerebellar artery; RAG = radial artery graft; SAH = subarachnoid hemorrhage; SCA = superior cerebellar artery; STA = superficial temporal artery; SVG = saphenous vein graft; VA = vertebral artery; VADA = VA dissecting aneurysm.

SUBMITTED February 15, 2015. ACCEPTED August 6, 2015.

INCLUDE WHEN CITING Published online February 5, 2016; DOI: 10.3171/2015.8.JNS15362. 
Concurrently, there has been a decline in open surgery for VADAs because of the difficulty of treating these aneurysms. However, recent technical and instrumental improvements for vascular reconstruction,,$^{17,20,21,41}$ as well as advances in skull base techniques, ${ }^{14,15,26,27}$ have again made open surgery a viable option for treating these complex aneurysms. This study describes the surgical strategy for the radical treatment of bilateral VADAs using bypass surgery.

\section{Methods}

Between April 2010 and April 2014, 7 patients with bilateral VADAs were treated. One patient who had undergone coil embolization performed at another hospital after SAH presented to our institution with progressive enlargement of the contralateral VA. One patient who had undergone coil embolization at an outside facility presented to our service with a recurrent aneurysm. Another patient, who was initially treated at another hospital with VA distal clipping and occlusion, presented to us with enlargement of the aneurysm and enlargement of the contralateral VADA. The 4 other cases presented without previous treatment with progressive enlargement of the aneurysms. Every patient underwent full clinical and neurological evaluations performed by an independent senior consultant neurosurgeon before treatment and at awakening, discharge, and the 6-month follow-up. The modified Rankin Scale (mRS) scores were recorded.

\section{Surgical Intervention}

The surgical indications for VADA included SAH, enlargement of the aneurysm, partial aneurysm thrombosis, and brainstem compression. Our surgical algorithm was as follows. Patients in whom 1 VA was occluded underwent surgical or endovascular $\mathrm{V}_{3}$-posterior cerebral artery (PCA) anastomosis using a radial artery graft (RAG) or a saphenous vein graft (SVG) with proximal occlusion or trapping of the affected VA. If the posterior inferior cerebellar artery (PICA) arose from the aneurysm or at the trapping site, an occipital artery (OA)-PICA anastomosis was performed. In cases of bilateral VA involvement, a 2 -stage operation was performed. In the first operation, trapping of the VA with reconstruction, such as a $V_{3}-V_{4}$ or $\mathrm{V}_{4}-\mathrm{V}_{4}$ bypass using RAG or $\mathrm{SVG}$, of the VA on 1 side was performed. If reconstruction of the VA was difficult, or dome clipping with preservation of VA anterograde blood flow was easy to perform, dome clipping with preservation of VA anterograde blood flow was selected. Then, proximal occlusion or trapping of the contralateral VA was performed in the second operation (Fig. 1).

\section{Results}

The clinical characteristics of the 7 patients are presented in Table 1 . There was a predominance of male patients $(71.4 \%)$. The ages of these patients ranged from 25 to 63 years (mean age 50.2 years). Perioperative complications were encountered in 3 patients during the first operation and in 1 patient during the second operation. The clinical presentation was sudden onset of posterior cervical pain in 4 patients, severe headache in 2 patients, and SAH in 1 patient (Hunt and Kosnik Grade II). The mean clinical follow-up was 19.2 months, and the mean angiographic follow-up was 18.4 months. Transient complications after the first operation were observed in 2 cases: 1 patient experienced facial nerve palsy and dysphagia, and 1 patient had severe dysphagia. It was difficult to discern

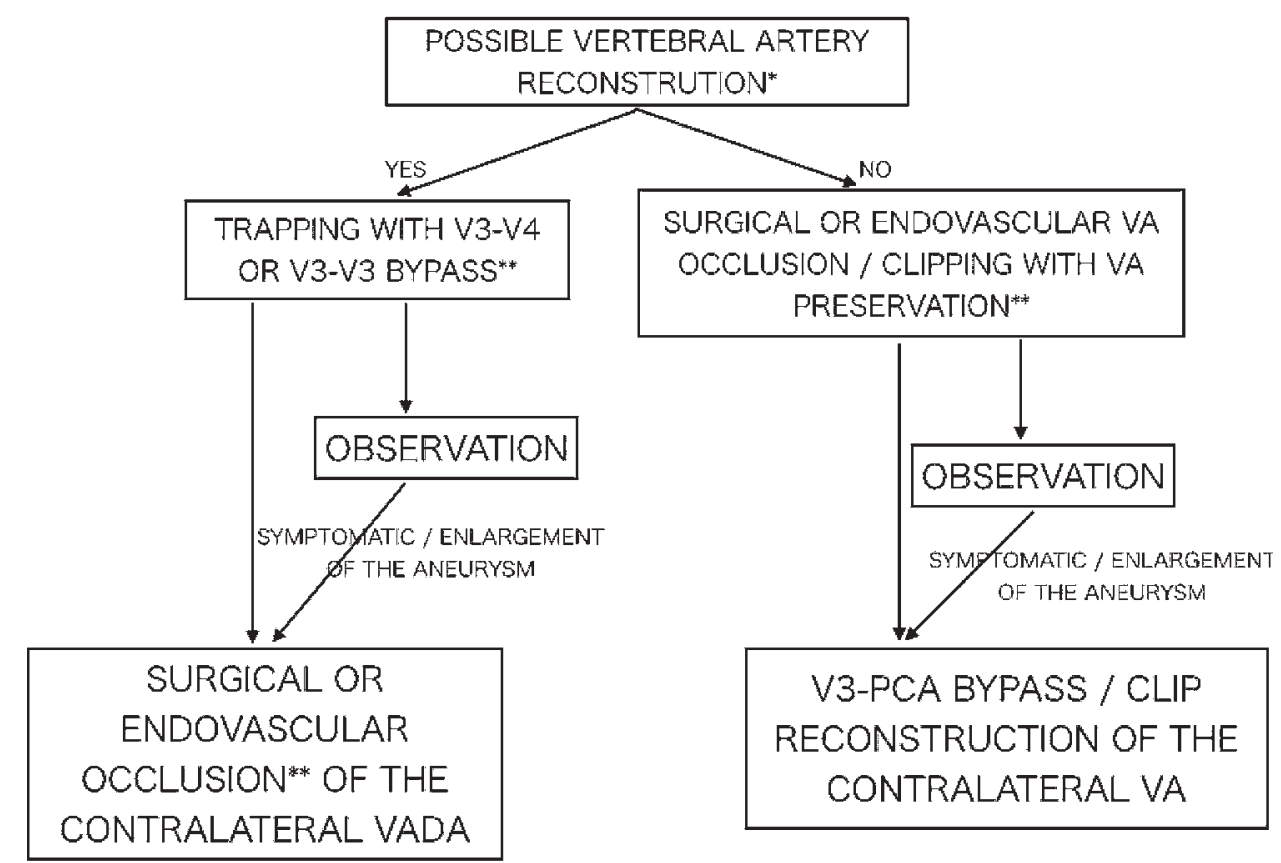

FIG. 1. Flowchart of the surgical strategy for bilateral VADAs. *The first interventional side should be determined as follows: for unruptured VADAs, whichever side is easier for reconstruction should be treated first; for ruptured bilateral VADA, the ruptured side should be treated first. ${ }^{* *}$ OA-PICA bypass or another bypass should be combined if needed. 

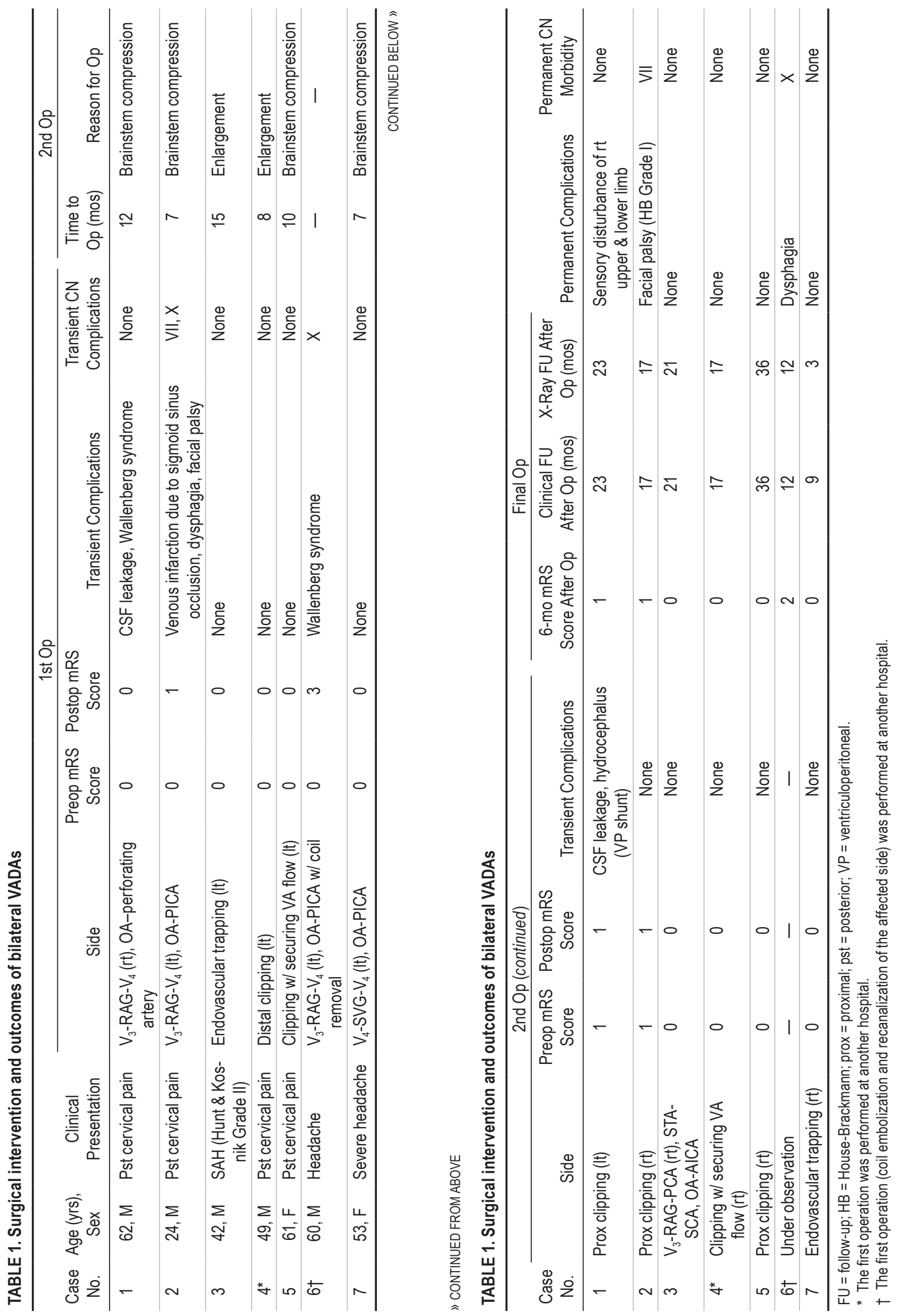
whether these 2 complications were a result of cranial nerve $(\mathrm{CN})$ disturbance or brainstem disturbance since the former patient suffered a petrosal vein infarction and the latter patient suffered a lateral medullary infarction. The patient in Case 1 developed Wallenberg syndrome on the reconstructed side 1 month after the operation. There was only 1 transient complication (CSF leakage) after the second operation. There were no cases of mRS score worsening between the first and second operations. The $\mathrm{mRS}$ scores at 6 months after the final operation were 0 in 4 patients, 1 in 2 patients, and 2 in 1 patient. Permanent morbidity consisted of 1 case of sensory disturbance due to Wallenberg syndrome, 1 case of mild facial nerve palsy (House-Brackmann Grade I), and 1 case of dysphagia. No recurrence or other complications were identified during the follow-up.

\section{Illustrative Cases \\ Case 2}

A 24-year-old man developed sudden occipital neck pain and presented to our hospital where he was diagnosed with bilateral VADAs. Computed tomography angiography (CTA) showed fusiform aneurysms of the bilateral VAs. The left-side aneurysm was fusiform and partially thrombosed (Fig. 2).
The first operation was performed on the left side because the bilateral VAs deviated to the left side. This meant that the distal $\mathrm{V}_{4}$ portion of the VA was easy to anastomose. The operation was performed in the parkbench position with a $\mathrm{C}$-shaped skin incision. The suboccipital muscle dissection and transcondylar fossa approach were performed, followed by an OA-PICA bypass (the occlusion time was 25 minutes and 34 seconds). The aneurysm was then trapped and removed. We then proceeded with a RAG-V $\mathrm{V}_{4}$ bypass superior to the $\mathrm{CN}$ VII/VIII complex and then a $\mathrm{V}_{3}-\mathrm{RAG}$ anastomosis (Figs. 3 and 4). The total reconstruction time for $\mathrm{V}_{3}-\mathrm{RAG}-\mathrm{V}_{4}$ bypass was 65 minutes. The anterograde flow of both bypasses was good. Postoperative CTA showed good filling of the anterograde flow in the left VA. There was no immediate postoperative neurological deficit, but 24 hours after surgery the patient become drowsy and developed left CN VII palsy. CT showed obstructive hydrocephalus and hemorrhagic venous infarction in the petrosal vein territory. CT venography showed that the sigmoid sinus and petrosal vein were thrombosed. An external ventricular drain was placed, and the patient was managed medically. The facial palsy and auditory disturbance improved gradually, and the patient was discharged with a mRS score of 1 .

The second operation was performed 6 months after the first. Proximal clipping was performed on the right
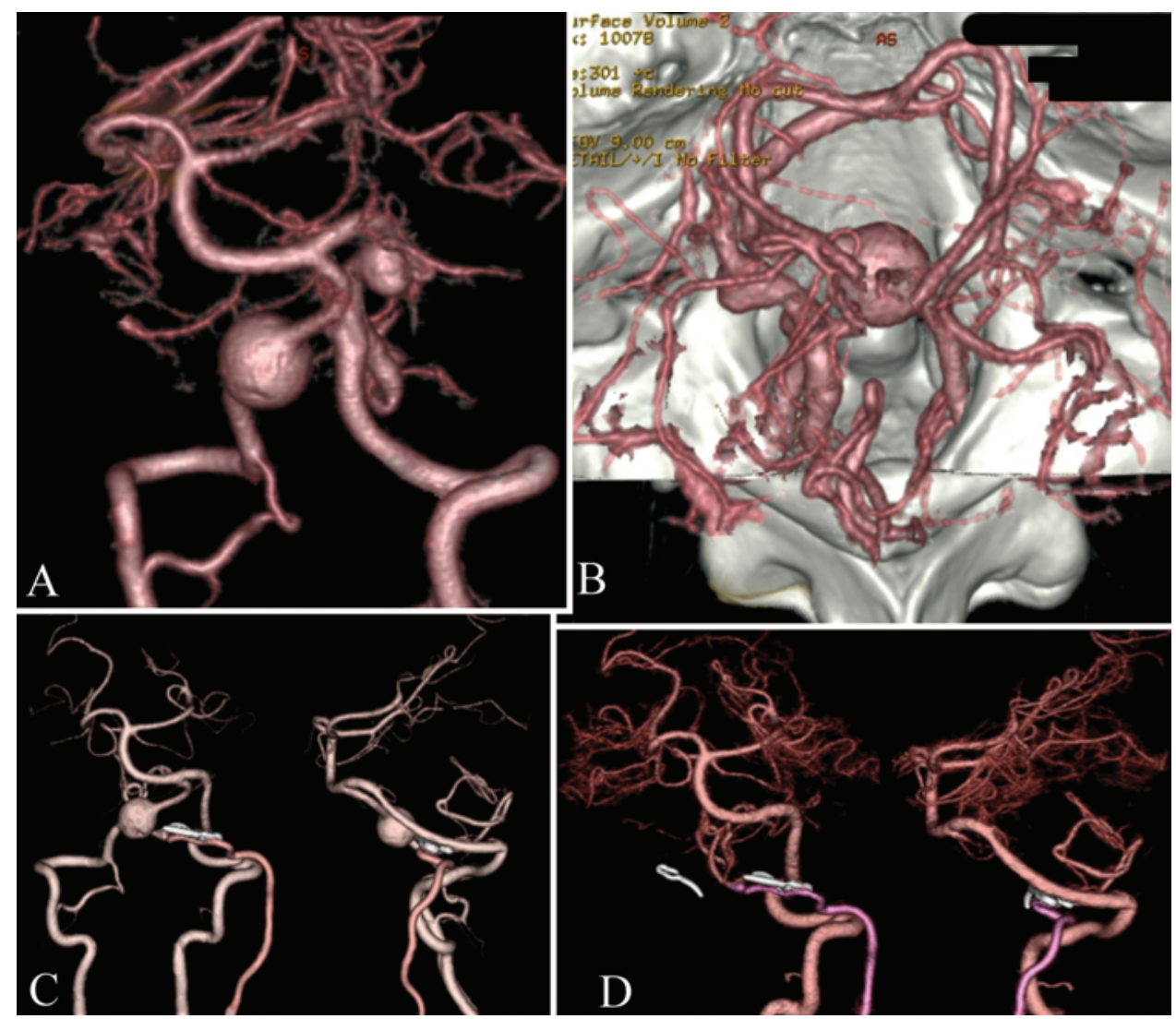

FIG. 2. Case 2. A: Preoperative CT angiogram showing bilateral VADAs and the partial thrombosis of the left-sided aneurysm. B: The bilateral VAs deviate strongly to the left side. The left-sided PICA also shows fusiform aneurysmal formation. C: Postoperative CT angiogram obtained after the first operation, showing good filling of the reconstructed VA and the OA-PICA anastomosis. D: Postoperative CT angiogram obtained after the second operation, showing a diminished left-sided aneurysm and good anterograde flow of the reconstructed left-sided VA. Figure is available in color online only. 

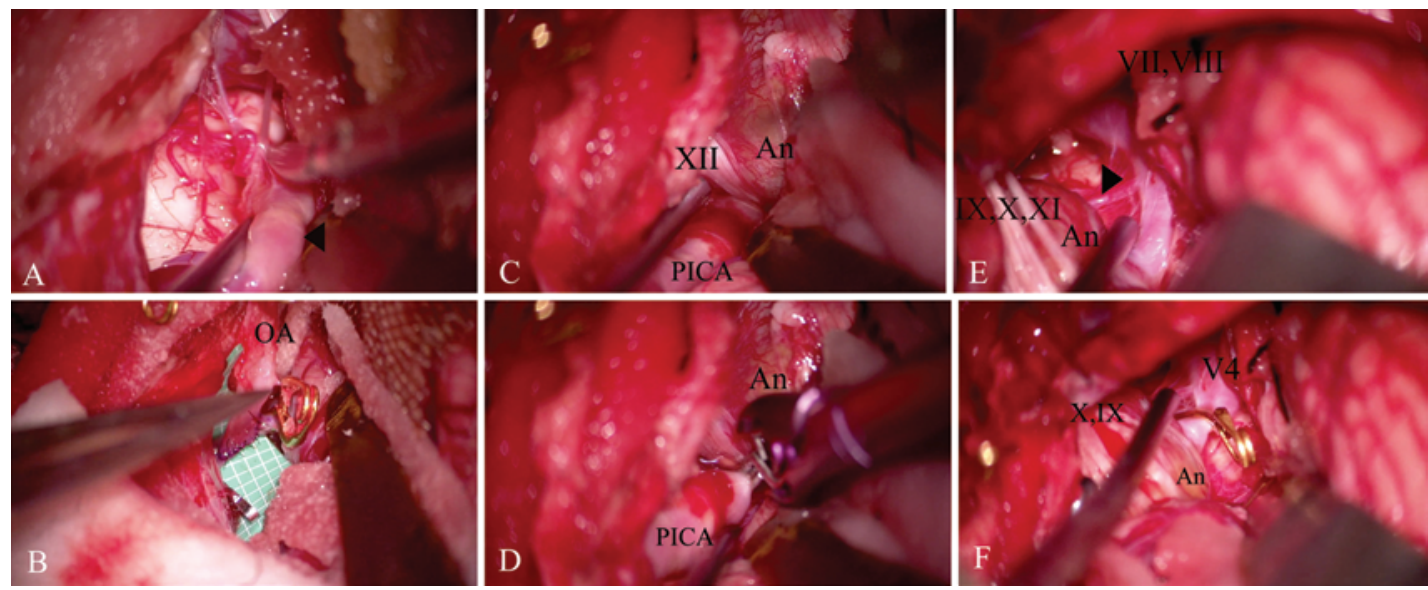

FIG. 3. Case 2. Intraoperative images of the left-side transcondylar fossa approach. A: The PICA shows fusiform aneurysmal formation (arrowhead). B: OA-PICA anastomosis was performed. The anastomosis site of the PICA was not on the pathological wall, but on the normal wall. C: The proximal VA is secured caudal to the hypoglossal nerve. D: The proximal VA is clipped. E: The distal VA is secured in between CN IX, the CN X/XI complex, and the CN VII/VIII complex. The anterior spinal artery is shown (arrowhead). F: The distal VA is clipped proximal to the anterior spinal artery. An = aneurysm. Figure is available in color online only.

side. The patient's postoperative course was without complications. The final CTA showed good filling of the left VA with no aneurysm.

\section{Case 3}

A 42-year-old man developed an SAH (Hunt and Kosnik Grade II) and was transferred to another hospital where bilateral VADAs with left-sided rupture were diagnosed. Endovascular trapping of the right VA was performed, and he was discharged with an $\mathrm{mRS}$ score of 0 . However, at his 3-month follow-up, the contralateral aneurysm was noted to be growing, and the patient's care was transferred to our institution (Fig. 5).

A $\mathrm{V}_{3}$-RAG-PCA bypass was planned (Figs. 6 and 7). The patient was placed in a left-side park-bench position, and suboccipital muscle dissection was performed with harvesting of the OA. The parietal branch and frontal branch of the superficial temporal artery (STA) were also harvested. Suboccipital craniotomy, temporal craniotomy, and mastoidectomy along with a transcondylar fossa ap-
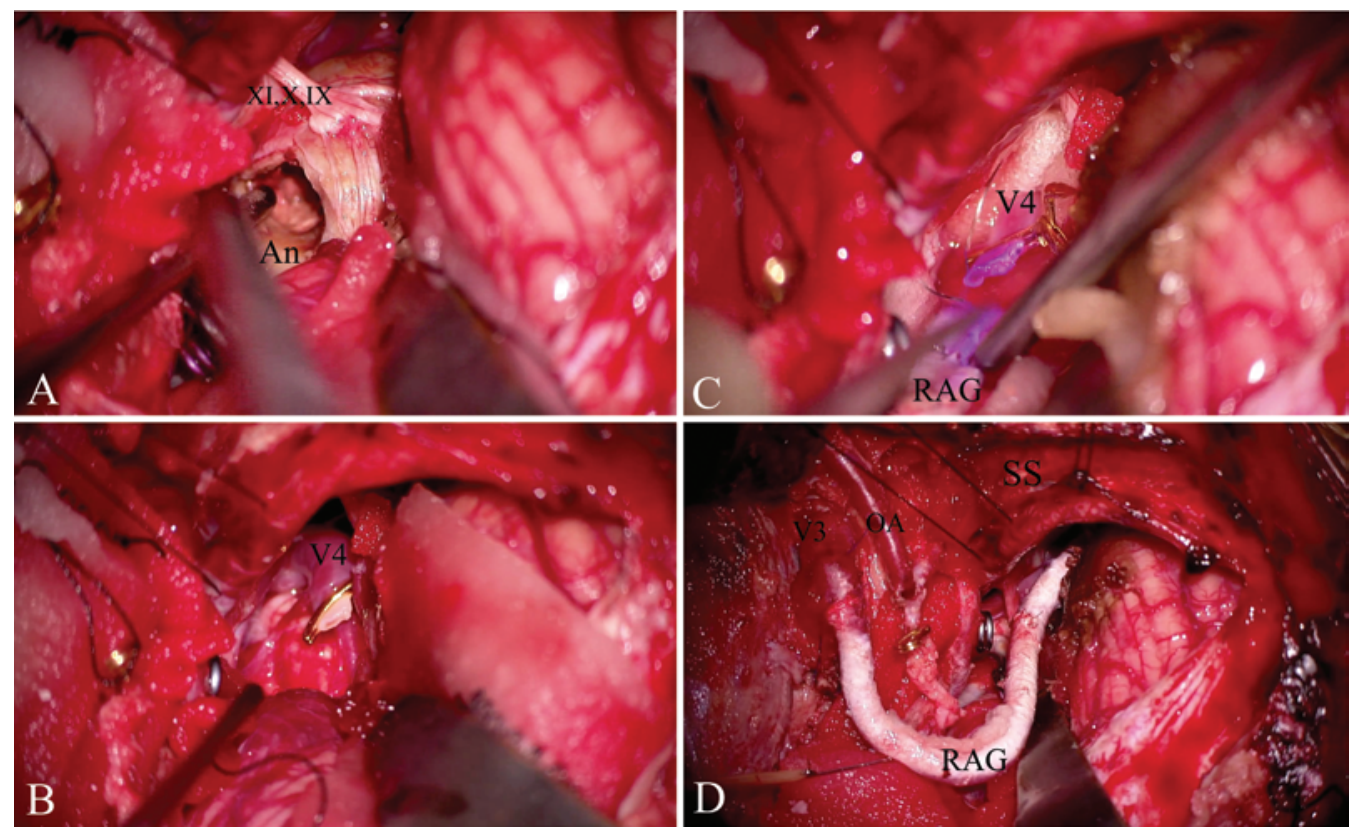

FIG. 4. Case 2. Intraoperative images of the transcondylar fossa approach on the left side. A: After trapping the aneurysm, thrombectomy is performed. B: After internal decompression of the aneurysm, the aneurysm is removed. There is a wide space

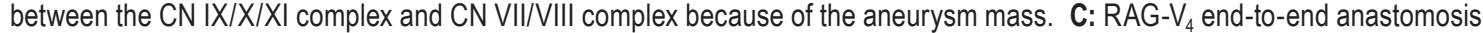
is performed. D: Then, $V_{3}$-RAG side-to-end anastomosis is performed. Here, the reconstruction of the VA is established. SS = sigmoid sinus. Figure is available in color online only. 

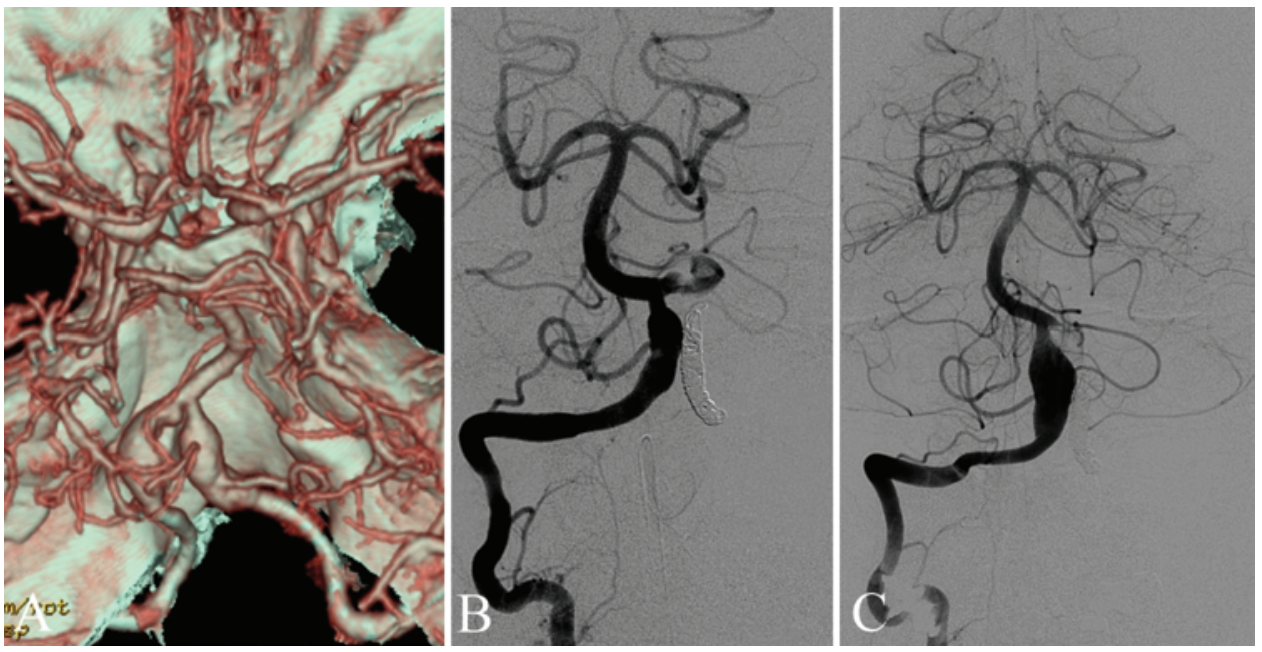

FIG. 5. Case 3. A: Preoperative CTA was performed when the patient suffered a subarachnoid hemorrhage. The bilateral VADAs are shown. B: Left-side endovascular trapping is performed. C: Digital subtraction angiography performed 3 months after trapping shows enlargement of the right-side VADA. Figure is available in color online only.

A

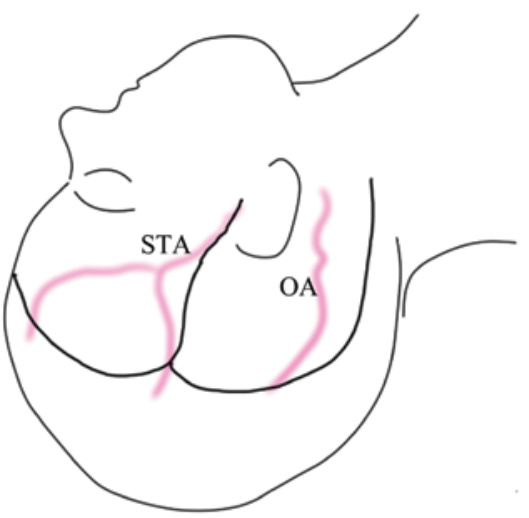

A

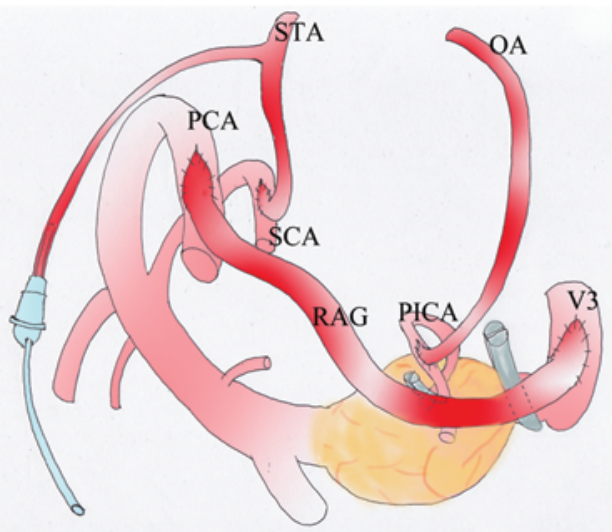

C pressure monitoring

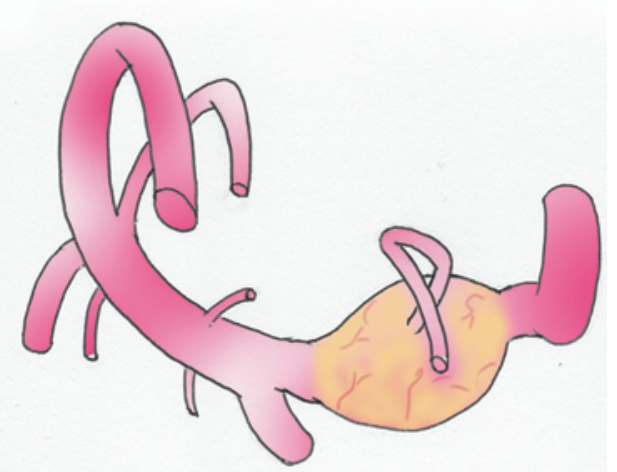

B

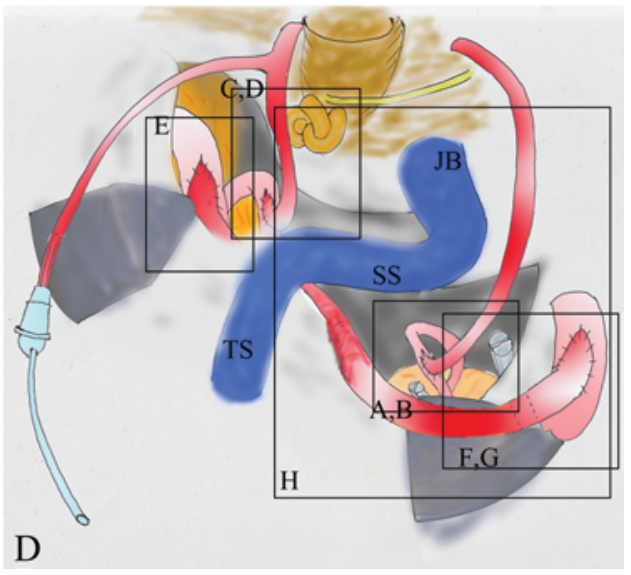

FIG. 6. Case 3. A: The skin incision was designed for just above the parietal branch of the STA, curved to the front midline, and then curved to the posterior neck to include the OA (black line). B: Schema of the posterior circulation and aneurysm. C: Schema of the final situation: proximal clipping of the right VA and clipping of the origin of the PICA with STA-SCA bypass, OA-PICA bypass, $V_{3}$-RAG-PCA bypass, and pressure monitoring from the STA. D: Schema for correlation with Fig. 7. The rectangular areas and letters indicate the view of the operative images shown in Fig. 7. JB = jugular bulb; TS = transverse sinus. Copyright Nakao Ota. Published with permission. Figure is available in color online only. 

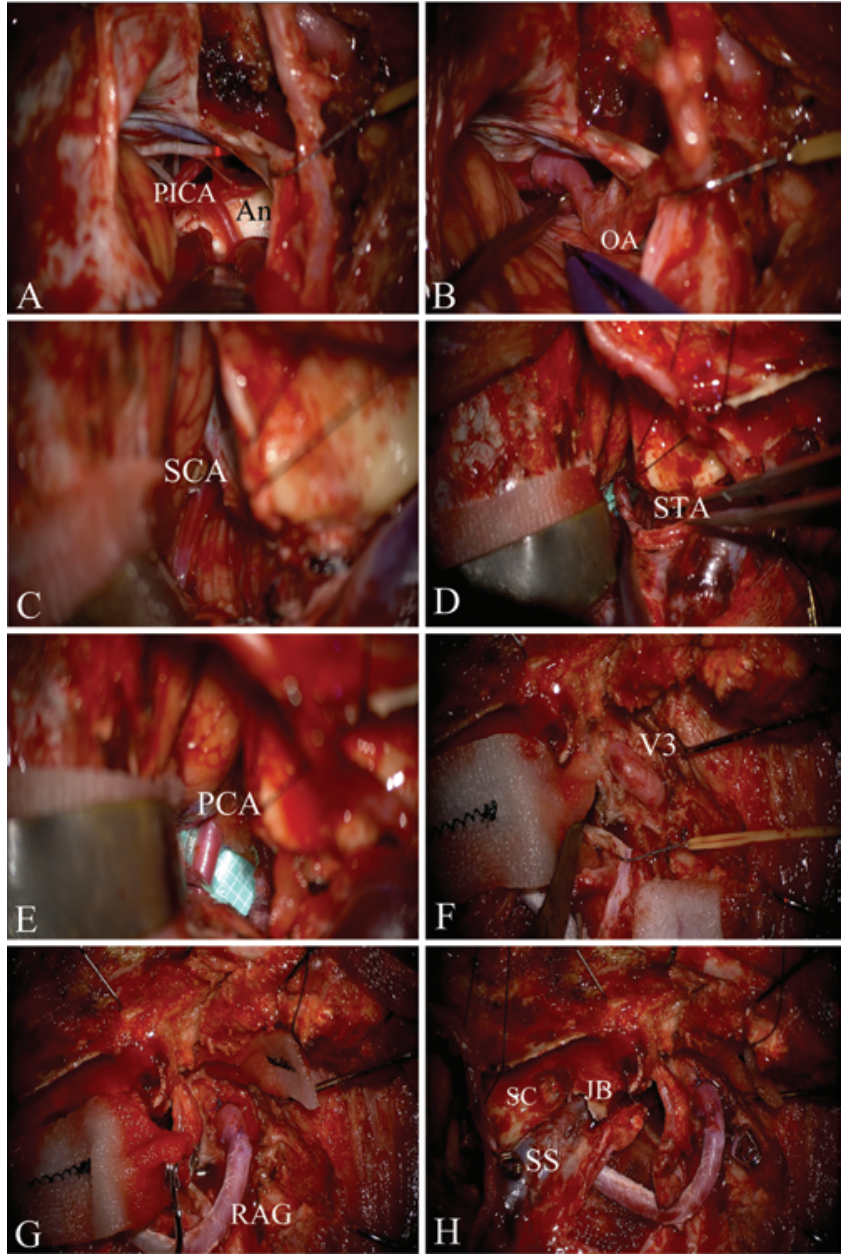

FIG. 7. Case 3. Intraoperative images of the approach on the right side. A: Aneurysm and PICA are determined from the retrosigmoid field. B: OA-PICA end-to-side anastomosis is performed. C: The SCA on the right is determined from the presigmoid space. D: STA-SCA anastomosis is performed. $E$ : The right $P_{2}$ portion of the PCA is determined from the presigmoid space. The PCA is present more in the cranial direction than the SCA. Then, RAG-PCA end-to-side anastomosis is performed. $F$ : The $V_{3}$ portion of the $V A$ is determined. $G: V_{3}-R A G$ side-to-end anastomosis is performed. $\mathrm{H}$ : The RAG is running from the retrosigmoid space to the presigmoid space. $\mathrm{SC}=$ semicircular canal. Figure is available in color online only.

proach were performed. First, OA-PICA bypass was performed (the occlusion time was 24 minutes and 2 seconds), and then STA-SCA bypass was performed as a backup during VA occlusion (the occlusion time was 32 minutes and 3 seconds). Next, a $\mathrm{V}_{3}$-RAG-PCA bypass was performed. The occlusion time of the RAG-PCA anastomosis was 45 minutes and 17 seconds, and that of $\mathrm{V}_{3}$-RAG anastomosis was 12 minutes and 9 seconds. Pressure monitoring performed on the other branch of the STA-SCA bypass showed a decreased pressure wave when the $\mathrm{V}_{3}$ portion of the VA was temporarily occluded. After establishing $\mathrm{V}_{3^{-}}$ RAG-PCA bypass, the pressure improved, thus indicating that the $\mathrm{V}_{3}$-PCA bypass was completely achieved. Finally, proximal clipping of the right VA and the origin of the PICA was performed. Postoperative CTA showed good filling of the $\mathrm{V}_{3}$-RAG-PCA, STA-SCA, and OA-PICA anastomoses without any aneurysm filling (Fig. 8). The patient was discharged with an mRS score of 0 .

\section{Case 4}

A 49-year-old man developed sudden headache and was diagnosed with bilateral VADAs. He was initially followed conservatively. During this period of observation, the VADA on the left side grew and was treated by distal VA clipping/occlusion at another institution (Fig. 9). Follow-up at 1 year later showed that the contralateral VA was growing. He then came to our hospital.

A $V_{3}$-RAG-PCA bypass with VA proximal occlusion was planned. First, the transcondylar fossa approach was performed, and the aneurysm and parent artery were identified. The aneurysm dome was easily identified, making clipping of the aneurysm while securing VA blood flow technically feasible (Fig. 10). The patient was discharged with an mRS score of 0 and is undergoing periodic followup by CTA.

\section{Discussion}

The best treatment for unilateral VADA remains parent artery occlusion, ${ }^{10,12,22,34}$ but it is not feasible for bilateral VADAs. Trapping the VA places increased hemodynamic stress on the contralateral VA and leads to enlargement and possibly rupture. ${ }^{11,13,36}$ The same findings were seen in this series.

Although endovascular treatment for bilateral VADAs, such as stent-assisted coiling, the stent-within-a-stent technique, and covered stent graft, have been reported to have good outcomes, ${ }^{1,8,19,38,42,44}$ there are still concerns regarding their widespread use. First, the mass effect of the aneurysm cannot be decreased, especially in the early phase. Though Halbach et al. ${ }^{7}$ reported on the efficacy of endovascular embolization of intracranial aneurysms in patients presenting with a mass effect, many reports suggest that aneurysm enlargement occurs after endovascular trapping. $2,3,9,23,39$ This means that compression of the brainstem may increase and neurological symptoms may worsen. Second, the long-term outcomes are unclear. Iihara et al. ${ }^{9}$ reported the continued enlargement of a thrombosed VA aneurysm after complete endovascular trapping. They reported that adventitial neovascularization by the vasa vasorum may play a key role in enlargement, and surgical clip placement and aneurysmectomy should be considered. Thus, patients who were treated with endovascular trapping with a well-developed vasa vasorum require longterm follow-up. If recurrence or enlargement occurs, surgical trapping and aneurysmectomy are needed..$^{30,37}$ Third, patients who undergo stenting need continued antiplatelet agents and strict follow-up using digital subtraction angiography. Lastly, the covered stent should avoid the orifice of the PICA, even when it is involved in the aneurysm. Thus, the aneurysmal wall with the involved vasa vasorum may not be completely occluded, and there may be a risk of recurrence and regrowth.

Flow-diverting stents have recently been introduced (these devices are not approved in Japan as of March 2015), and the reported periprocedural and midterm follow-up results to date have been impressive $e^{5,6,24,40}$ and 

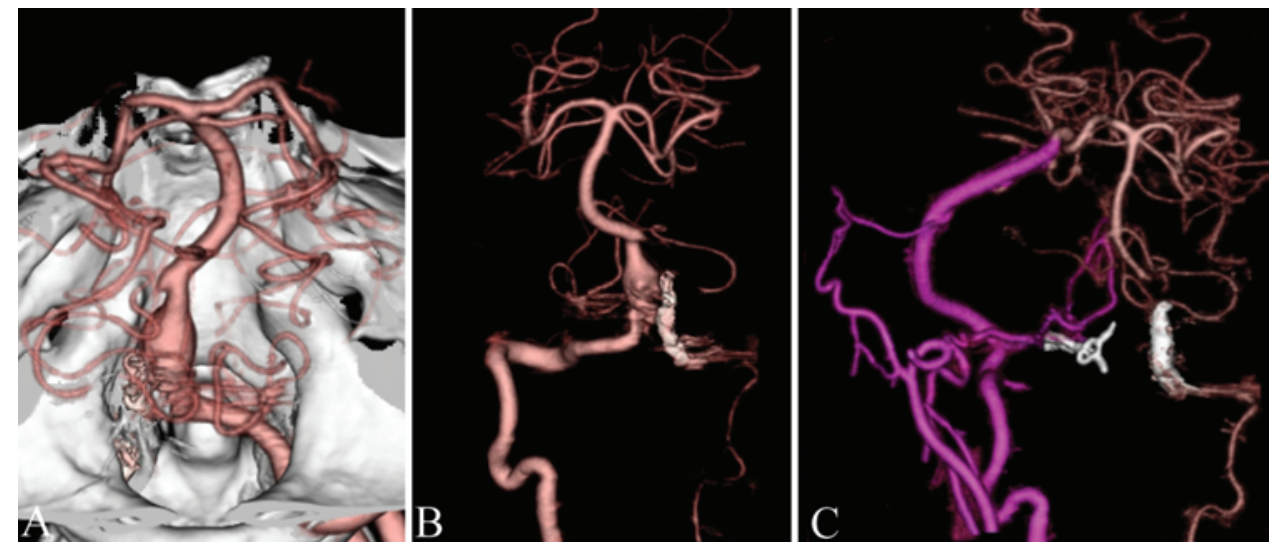

FIG. 8. Case 3. Pre- and postoperative CTA images. A: Preoperative CT angiogram showing the right-side VADA. The bilateral VAs deviate strongly to the left side. B: Preoperative CT angiogram. C: Postoperative CT angiogram showing good filling of the $V_{3}$-RAG-PCA bypass, STA-SCA bypass, and OA-PICA bypass. Figure is available in color online only.

marked by high rates of curative parent artery reconstruction with a reasonable level of treatment-associated morbidity and mortality. However, these studies include not only VADAs but also other aneurysm locations. Moreover, few studies focused on VADA; ${ }^{43}$ especially in terms of bilateral VADAs, there has been only 1 case report. ${ }^{28}$ In addition, flow-diverting stents are not approved for clinical application in Japan, and, as a very new technology, there are still few long-term follow-up results. ${ }^{43}$ The previously mentioned concerns about other endovascular devices also exist for flow-diverting stents. Fiorella et al. ${ }^{4}$ and Klisch et al. ${ }^{16}$ reported very late thrombosis and occlusion of the VA and basilar artery after stopping clopidogrel because they could not achieve complete aneurysm obliteration, and the aneurysmal mass was not decreased. Presently, it is unclear if the intima covered by the stent retains its normal function, and patients may have to continue antiplatelet agents almost permanently. Martin et al. ${ }^{25}$ reported delayed aneurysm rupture from thrombus-associated autolysis of the aneurysmal wall. Another important consideration is the maintenance of the patency of the branch vessels or perforators from the parent vessel. This is theoretically possible due to the presence of interstices between the stent strands, but the results have been mixed with cases of branch artery occlusion being reported. ${ }^{25}$ Hence, direct surgical treatment using bypass, while highly technically demanding, may confer several advantages over endovascular therapies. First, there is no need to continue dual antiplatelet therapy because the reconstructed VA is covered by the normal intima. Second, complete obliteration of the aneurysm by trapping can be performed, and mass reduction by aneurysmectomy and thrombectomy can be achieved. If aneurysmectomy can be accomplished, the risk of recurrence or regrowth is eliminated. Third, OA-PICA anastomosis is easily added if PICA is involved in the lesion. Finally, if one side of the bilateral VADAs is treated by VA reconstruction, the treatment of the contralateral VADA is only parent artery occlusion, which is known to provide reliable outcomes. . $^{10,12,22,34}$

Vascular reconstruction of the VA, ${ }^{20,21}$ such as $\mathrm{V}_{3}$-RAG$\mathrm{V}_{4}$ anastomosis as the initial operation, is the most useful microsurgical method of treating bilateral VADAs. Favor- able outcomes can be achieved if performed by a skilled neurosurgeon. Because the contralateral VA compensates for the blood flow of the basilar artery during anastomosis, there is sufficient time for temporary occlusion and reconstruction. After one VA is reconstructed, the treatment of the other side involves only parent artery occlusion. There are several essential components to this complex surgery. Determination of the initial operative side is key. Many cases of bilateral VADAs have serpentine-like VAs that deviate significantly laterally. The side to be reconstructed must be the one that is technically easier to reconstruct, even if the aneurysm on that side is smaller than the other. Dome clipping while securing VA blood flow is another option for these aneurysms if dome clipping appears feasible or VA reconstruction proves difficult. This may reduce lower $\mathrm{CN}$ injuries during the operation. However, this method also has problems, similar to endovascular stenting or trapping, and the pathologically abnormal vessel wall involving the vasa vasorum remains. This means that

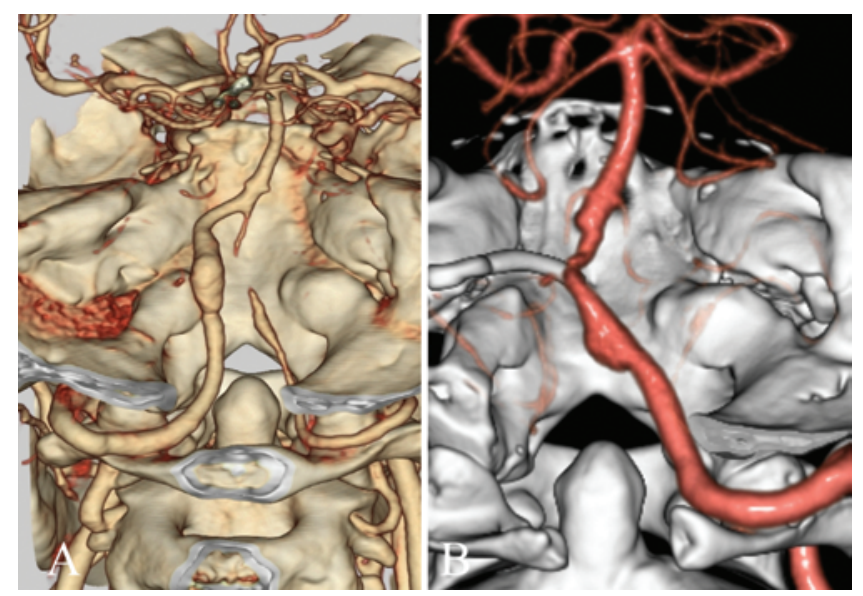

FIG. 9. Case 4. Pre- and postoperative CTA images of the first operation. A: Before the first operation, the CT angiogram shows bilateral VADAs. The left side shows fusiform dilation, and the right side shows the string sign with a thrombosed aneurysm. B: After the first operation on the left side, CTA shows enlargement of the right-side aneurysm. Figure is available in color online only. 

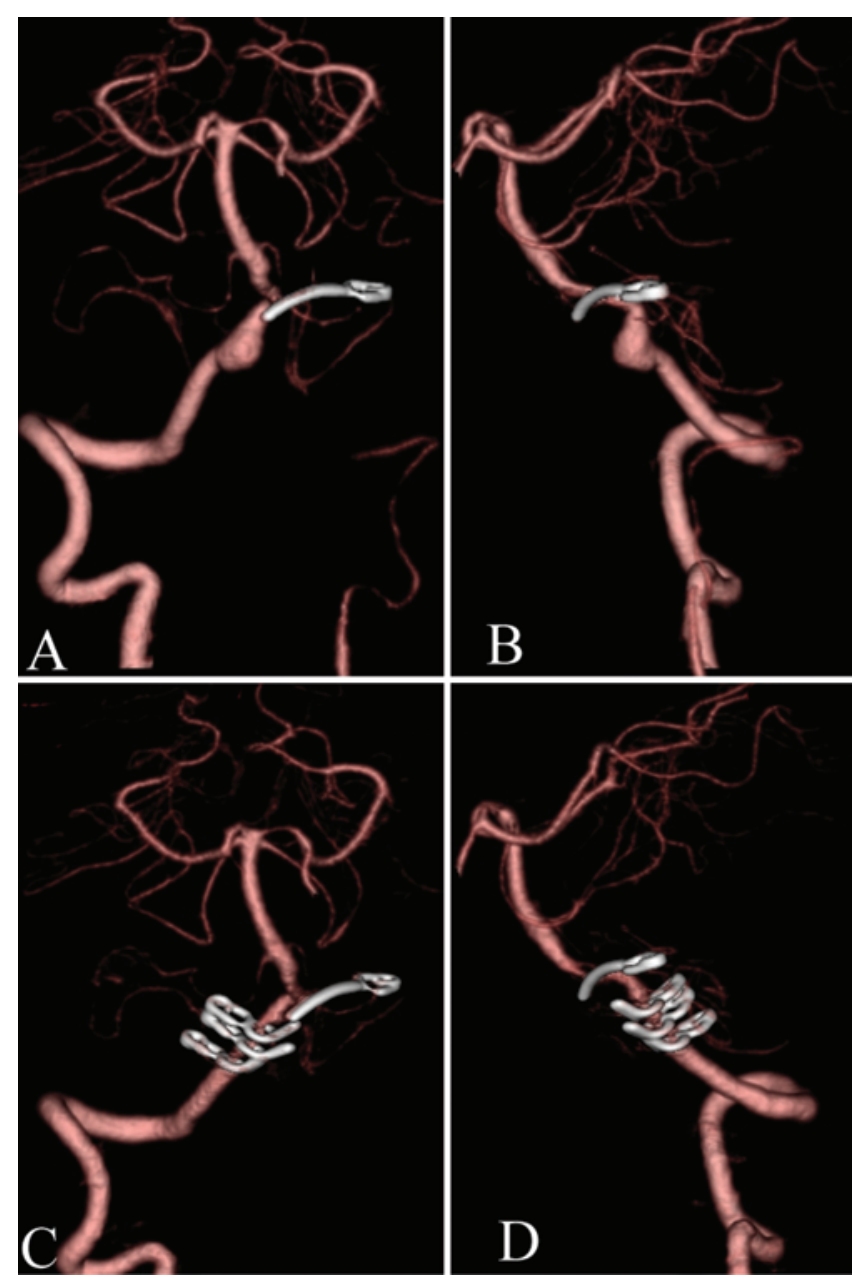

FIG. 10. Case 4. Pre- and postoperative CTA images of the second operation. A: Anterior to posterior CTA images before the second operation. B: Lateral view from left side before the second operation. C: Anteroposterior view after the second operation, showing how dome clipping with securing of the VA anterograde blood flow was performed. D: Lateral view from the left side after the second operation. Figure is available in color online only.

there is a risk of recurrence or regrowth of the aneurysm, and such cases require strict observation.

Though there are some case reports of favorable outcomes with trapping the ruptured side and waiting for the spontaneous resolution of the contralateral unruptured dissecting aneurysm ${ }^{31,32}$ or using bilateral conservative treatment, ${ }^{29}$ long-term outcomes are still unknown and patients should be followed carefully. Staged bilateral occlusion for vertebrobasilar VADAs has been reported ${ }^{11,35}$ as having a favorable outcome, but it cannot be tolerated in many cases of bilateral VADAs if the patients do not have sufficient flow from the posterior communicating artery. Thus, there is a high risk of residual VA occlusion if the posterior communicating artery is not well developed. Hence, in cases where one side of the VA is occluded and the other side grows or ruptures (Case 3 in the present series), a $\mathrm{V}_{3}$-PCA bypass must be performed before VA trapping. However, $\mathrm{V}_{3}$-PCA bypass in this situation carries some risk because the blood flow of the basilar artery is stopped during anas- tomosis of the $\mathrm{V}_{3}$ portion of the VA; the contralateral VA is occluded and cannot compensate for the blood flow of the basilar artery. Thus, before performing $\mathrm{V}_{3}$-PCA bypass, a backup bypass - such as STA-superior cerebellar artery (SCA), STA-anterior inferior cerebellar artery (AICA), or OA-AICA bypass - is needed to provide adequate blood flow during anastomosis. Since malfunction of the $\mathrm{V}_{3}$ PCA is fatal, pressure monitoring from the other branch of these backup bypasses should be performed (Case 3 in the present series).

The position for this approach is the park-bench position, and suboccipital muscle dissection is necessary. Suboccipital muscle dissection allows easy harvesting of the occipital artery and securing of the $\mathrm{V}_{3}$ portion of the VA. The most important merit of suboccipital muscle dissection is that the operative field becomes shallower and wider than the 1-layer approach, ${ }^{14}$ which facilitates less cumbersome suturing of the $\mathrm{V}_{3}-\mathrm{V}_{4}$ anastomosis.

A transcondylar fossa approach ${ }^{15,26,27}$ is needed for a $V_{3^{-}}$ $\mathrm{V}_{4}$ bypass, or a combined presigmoid approach with mastoidectomy is needed for $\mathrm{V}_{3}$-PCA bypass. ${ }^{41}$ With the transcondylar fossa approach, one can look up the operative field and use eggshell drilling to expose the sigmoid sinus and, thus, make a wide operative field on the lateral side.

The suturing location of $\mathrm{V}_{4}$ anastomosis should be inferior to the CN VII/VIII complex, in between the CN VII/VIII complex and CN IX, or below CN X. The site of the end-to-end anastomosis between RAG and the distal end of the $\mathrm{V}_{4}$ segment of the VA may be determined after removing the aneurysm. This may be especially true for cases of large aneurysms where removal provides an appropriate space to perform the end-to-end anastomosis with RAG. If the distal side of the $\mathrm{V}_{4}$ segment could be mobilized superficially, end-to-end anastomosis becomes easier on the lower CNs.

\section{Conclusions}

Bilateral VADAs are treatable with favorable outcomes if direct VA reconstruction by RAG is performed with the correct operative strategy by a skilled neurosurgeon. A lateral suboccipital craniotomy provides sufficient exposure to perform the procedure. Suboccipital muscular dissection makes the operative field shallow and the operation easier to perform. Meticulous sigmoid sinus skeletonization and condylar fossa drilling are also important in order to widen the surgical field and perform the anastomosis in a deep surgical corridor in a layer-by-layer fashion.

\section{Acknowledgments}

We would like to thank Dr. Tarik Ibrahim and Dr. Felix Göhre for critically revising the manuscript.

\section{References}

1. Ahn JY, Han IB, Kim TG, Yoon PH, Lee YJ, Lee BH, et al: Endovascular treatment of intracranial vertebral artery dissections with stent placement or stent-assisted coiling. AJNR Am J Neuroradiol 27:1514-1520, 2006

2. Batjer HH, Purdy PD: Enlarging thrombosed aneurysm of the distal basilar artery. Neurosurgery 26:695-700, 1990

3. Chang SD, Marks MP, Steinberg GK: Recanalization and 
rupture of a giant vertebral artery aneurysm after hunterian ligation: case report. Neurosurgery 44:1117-1121, 1999

4. Fiorella D, Hsu D, Woo HH, Tarr RW, Nelson PK: Very late thrombosis of a pipeline embolization device construct: case report. Neurosurgery 67:onsE313-onsE314, 2010

5. Fiorella D, Kelly ME, Albuquerque FC, Nelson PK: Curative reconstruction of a giant midbasilar trunk aneurysm with the pipeline embolization device. Neurosurgery 64:212-217, 2009

6. Fiorella D, Woo HH, Albuquerque FC, Nelson PK: Definitive reconstruction of circumferential, fusiform intracranial aneurysms with the pipeline embolization device. Neurosurgery 62:1115-1121, 2008

7. Halbach VV, Higashida RT, Dowd CF, Barnwell SL, Fraser KW, Smith TP, et al: The efficacy of endosaccular aneurysm occlusion in alleviating neurological deficits produced by mass effect. J Neurosurg 80:659-666, 1994

8. He M, Zhang H, Lei D, Mao BY, You C, Xie XD, et al: Application of covered stent grafts for intracranial vertebral artery dissecting aneurysms. J Neurosurg 110:418-426, 2009

9. Iihara K, Murao K, Sakai N, Soeda A, Ishibashi-Ueda H, Yutani C, et al: Continued growth of and increased symptoms from a thrombosed giant aneurysm of the vertebral artery after complete endovascular occlusion and trapping: the role of vasa vasorum. Case report. J Neurosurg 98:407-413, 2003

10. Iihara K, Sakai N, Murao K, Sakai H, Higashi T, Kogure S, et al: Dissecting aneurysms of the vertebral artery: a management strategy. J Neurosurg 97:259-267, 2002

11. Inoue A, Kohno K, Takechi A, Kohno K, Matsushige T, Takeda T: Bilateral vertebral artery dissecting aneurysm with subarachnoid hemorrhage treated with staged bilateral vertebral artery coil occlusion: a case report. Surg Neurol 70:319-322, 2008

12. Kakino S, Ogasawara K, Kubo Y, Otawara Y, Tomizuka N, Suzuki M, et al: Treatment of vertebral artery aneurysms with posterior inferior cerebellar artery-posterior inferior cerebellar artery anastomosis combined with parent artery occlusion. Surg Neurol 61:185-189, 2004

13. Katsuno M, Mizunari T, Kobayashi S, Takahashi H, Teramoto A: Rupture of a vertebral artery dissecting aneurysm developing immediately after trapping of a dissecting aneurysm on the contralateral vertebral artery: case report. Neurol Med Chir (Tokyo) 49:468-470, 2009

14. Katsuno M, Tanikawa R, Uemori G, Kawasaki K, Izumi N, Hashimoto M: Occipital artery-to-posterior inferior cerebellar artery anastomosis with multiple-layer dissection of suboccipital muscles under a reverse C-shaped skin incision. $\mathbf{B r}$ J Neurosurg 29:401-405, 2015

15. Kawashima M, Matsushima T, Inoue T, Mineta T, Masuoka J, Hirakawa N: Microvascular decompression for glossopharyngeal neuralgia through the transcondylar fossa (supracondylar transjugular tubercle) approach. Neurosurgery 66 (6 Suppl Operative):275-280, 2010

16. Klisch J, Turk A, Turner R, Woo HH, Fiorella D: Very late thrombosis of flow-diverting constructs after the treatment of large fusiform posterior circulation aneurysms. AJNR Am J Neuroradiol 32:627-632, 2011

17. Kobayashi N, Hiroyasu K: Reconstruction of the basilar arterial system: Methods of high-flow bypass in posterior cranial fossa. Surg Cereb Stroke (Jpn) 27:270-276, 1999

18. Koh JS, Ryu CW, Lee SH, Bang JS, Kim GK: Bilateral vertebral-artery-dissecting aneurysm causing subarachnoid hemorrhage cured by staged endovascular reconstruction after occlusion. Cerebrovasc Dis 27:202-204, 2009

19. Kono K, Shintani A, Fujimoto T, Terada T: Stent-assisted coil embolization and computational fluid dynamics simulations of bilateral vertebral artery dissecting aneurysms presenting with subarachnoid hemorrhage: case report. Neurosurgery 71:E1192-E1201, 2012

20. Kubota H, Tanikawa R, Katsuno M, Izumi N, Noda K, Ota N, et al: Vertebral artery-to-vertebral artery bypass with interposed radial artery or occipital artery grafts: surgical technique and report of three cases. World Neurosurg 81:202. e1-202.e8, 2014

21. Kubota H, Tanikawa R, Katsuno M, Noda K, Ota N, Miyata $\mathrm{S}$, et al: Reconstruction of intracranial vertebral artery with radial artery and occipital artery grafts for fusiform intracranial vertebral aneurysm not amenable to endovascular treatment: technical note. Acta Neurochir (Wien) 155:15171524,2013

22. Kurata A, Ohmomo T, Miyasaka Y, Fujii K, Kan S, Kitahara $\mathrm{T}$ : Coil embolization for the treatment of ruptured dissecting vertebral aneurysms. AJNR Am J Neuroradiol 22:11-18, 2001

23. Lee KC, Joo JY, Lee KS, Shin YS: Recanalization of completely thrombosed giant aneurysm: case report. Surg Neurol 51:94-98, 1999

24. Lylyk P, Miranda C, Ceratto R, Ferrario A, Scrivano E, Luna $\mathrm{HR}$, et al: Curative endovascular reconstruction of cerebral aneurysms with the pipeline embolization device: the Buenos Aires experience. Neurosurgery 64:632-643, N6, 2009

25. Martin AR, Cruz JP, Matouk CC, Spears J, Marotta TR: The pipeline flow-diverting stent for exclusion of ruptured intracranial aneurysms with difficult morphologies. Neurosurgery 70 (1 Suppl Operative):21-28, 2012

26. Matsushima T, Fukui M: [Lateral approaches to the foramen magnum: with special reference to the transcondylar fossa approach and the transcondylar approach.] No Shinkei Geka 24:119-124, 1996 (Jpn)

27. Matsushima T, Kawashima M, Masuoka J, Mineta T, Inoue T: Transcondylar fossa (supracondylar transjugular tubercle) approach: anatomic basis for the approach, surgical procedures, and surgical experience. Skull Base 20:83-91, 2010

28. Mitchell B, Momin E, Jou LD, Shaltoni H, Morsi H, Mawad M: Extensive bilateral vertebral artery remodeling following treatment of dissection using pipeline embolic device. J Vasc Interv Neurol 7:5-8, 2014

29. Mizutani T, Aruga T, Kirino T, Miki Y, Saito I, Tsuchida $\mathrm{T}$ : Recurrent subarachnoid hemorrhage from untreated ruptured vertebrobasilar dissecting aneurysms. Neurosurgery 36:905-913, 1995

30. Nagahiro S, Takada A, Goto S, Kai Y, Ushio Y: Thrombosed growing giant aneurysms of the vertebral artery: growth mechanism and management. J Neurosurg 82:796-801, 1995

31. Nashimoto T, Saito T, Kurashima A, Yamashita S, Honma J: [Case of bilateral vertebral artery dissecting aneurysm presenting with subarachnoid hemorrhage.] No Shinkei Geka 37:381-385, 2009 (Jpn)

32. Nishimu YA, Ikeda A, Sugita R, Maeda K, Nakamua S, Takerroto M, et al: [Case of spontaneous bilateral VA dissecting aneurysm presenting with SAH.] No Shinkei Geka 35:583-589, 2007 (Jpn)

33. Otawara Y, Ogasawara K, Ogawa A, Kogure T: Dissecting aneurysms of the bilateral vertebral arteries with subarachnoid hemorrhage: report of three cases. Neurosurgery 50:1372-1375, 2002

34. Rabinov JD, Hellinger FR, Morris PP, Ogilvy CS, Putman CM: Endovascular management of vertebrobasilar dissecting aneurysms. AJNR Am J Neuroradiol 24:1421-1428, 2003

35. Sakamoto S, Ohba S, Shibukawa M, Kiura Y, Okazaki T, Arita K, et al: Staged bilateral vertebral artery occlusion for ruptured dissecting aneurysms of the basilar artery: a report of 2 cases. Surg Neurol 64:456-461, 2005

36. Somekawa K, Nagata K, Kawamoto S, Furuya H, Tanioka $\mathrm{D}$, Isoo A: [Treatment for the ruptured bilateral vertebral 
dissecting aneurysms.] No Shinkei Geka 30:321-325, 2002 (Jpn)

37. Sugita K, Kobayashi S, Takemae T, Tanaka Y, Okudera H, Ohsawa M: Giant aneurysms of the vertebral artery. Report of five cases. J Neurosurg 68:960-966, 1988

38. Suh SH, Kim BM, Park SI, Kim DI, Shin YS, Kim EJ, et al: Stent-assisted coil embolization followed by a stent-withina-stent technique for ruptured dissecting aneurysms of the intracranial vertebrobasilar artery. Clinical article. J Neurosurg 111:48-52, 2009

39. Swearingen B, Heros RC: Fatal rupture of a thrombosed giant basilar artery aneurysm. Surg Neurol 23:299-302, 1985

40. Szikora I, Berentei Z, Kulcsar Z, Marosfoi M, Vajda ZS, Lee $\mathrm{W}$, et al: Treatment of intracranial aneurysms by functional reconstruction of the parent artery: the Budapest experience with the pipeline embolization device. AJNR Am J Neuroradiol 31:1139-1147, 2010

41. Tanikawa R, Sugimura T, Hino K, Izumi N, Mitsui N, Yamauchi T, et al: Surgical application of skull base technique for EC-IC bypass to P2 segment. Surg Cereb Stroke (Jpn) 34:440-444, 2006

42. Wilkinson DA, Wilson TJ, Stetler WR, Pandey AS: Subarachnoid haemorrhage with bilateral intracranial vertebral artery dissecting aneurysms treated by staged endovascular stenting. BMJ Case Rep 2013:bcr0320126002, 2013

43. Yeung TW, Lai V, Lau HY, Poon WL, Tan CB, Wong YC: Long-term outcome of endovascular reconstruction with the Pipeline embolization device in the management of unruptured dissecting aneurysms of the intracranial vertebral artery. J Neurosurg 116:882-887, 2012

44. Yoon SM, Shim JJ, Kim SH, Chang JC: Bilateral vertebral artery dissecting aneurysms presenting with subarachnoid hemorrhage treated by staged coil trapping and covered stents graft. J Korean Neurosurg Soc 51:155-159, 2012

\section{Disclosures}

The authors report no conflict of interest concerning the materials or methods used in this study or the findings specified in this paper.

\section{Author Contributions}

Conception and design: Ota. Acquisition of data: Ota. Analysis and interpretation of data: Ota. Drafting the article: Ota. Reviewed submitted version of manuscript: Tanikawa. Approved the final version of the manuscript on behalf of all authors: Ota. Administrative/technical/material support: Eda, Matsumoto, Miyazaki, Matsukawa, Yanagisawa, Suzuki, Miyata, Oda, Noda, Tsuboi, Takeda, Kamiyama, Tokuda. Study supervision: Tanikawa, Takeda, Kamiyama, Tokuda.

\section{Supplemental Information}

\section{Previous Presentations}

This paper was presented at the 9th World Stroke Congress held in Istanbul, Turkey, October 22-25, 2014.

\section{Correspondence}

Nakao Ota, Department of Neurosurgery, Teishinkai Hospital, 1-6, Higashi 8, Kita 44, Higashi-ku, Sapporo, Hokkaido 0070844, Japan. email: nakao1980@gmail.com. 